\title{
Frequency Optimization in High Intensity Focused Ultrasound
}

\author{
Hasan Yetik*, Cemre Ariyurek ${ }^{\dagger}$, Ayhan Bozkurt ${ }_{*}^{\ddagger}$, and Arif Sanli Ergun* \\ TOBB University of Economics and Technology, Ankara, Turkey \\ Email: hyetik@etu.edu.tr \\ $\dagger$ Bilkent University, Ankara, Turkey \\ $\ddagger$ Sabanci University, Istanbul, Turkey
}

\begin{abstract}
In high intensity focused ultrasound (HIFU) the choice of transducer frequency depends on the target depth and tissue type. At high frequencies attenuation does not permit enough acoustical power to be transmitted to the target whereas at low frequencies the transmitted power is not absorbed efficiently. Hence, there exists an optimum frequency at which the power deposited at the target is maximum. In this study, we verified this relation experimentally using MR compatible focused transducers, ex-vivo tissue samples and magnetic resonance (MR) thermometry.
\end{abstract}

\section{INTRODUCTION}

High intensity focused ultrasound (HIFU) has been used in a number of therapeutic applications. It is generally accepted that HIFU has to be done at frequencies lower than the frequencies typically used for ultrasound imaging. The reason is that high acoustic attenuation in tissue at imaging frequencies does not allow the sufficient acoustic intensity to be delivered to the target location. Dual modality systems (imaging and therapy) suffer from this fact and are forced to compromise between therapy efficiency and image quality. A preferred solution to this is to use dual frequency/modality transducers [1]. Since acoustic radiation force is also proportional to the acoustic intensity at the target, same argument applies to acoustic radiation force imaging systems as well.

HIFU applications ranges from deep tissue thermal therapy where the target depth can be as large as $20 \mathrm{~cm}$ to small animal studies with target depths less than $1 \mathrm{~cm}$ to brain studies where the target lies behind a layer of bone. Because the efficacy of HIFU at the target is largely related to the frequency of the acoustic radiation, determining the frequency of operation and hence the frequency of the HIFU transducer is an important step [2]. Clearly, one single transducer will not be able to satisfy the needs of this broad range of HIFU applications. In this paper, we showed a simple analytical method to determine the optimum frequency for any specific target, and verified it experimentally using MR thermometry.

\section{ANALYTICAL BACKGROUND}

Acoustic intensity falls off exponentially in matter as a result of attenuation. For plane wave propagation acoustic intensity $I(z)$ at depth $z$ can be written as:

$$
I(z)=I_{0} e^{-2 \alpha_{0} f^{m} z}
$$

where $I_{0}$ is the acoustic intensity at $z=0, \alpha_{0}$ is the frequency independent attenuation coefficient, $f$ is the frequency and $m$ is the frequency dependence parameter. Power deposited at depth $z$ is then the product of the intensity at $z$ and the absorption coefficient at $z$ :

$$
P_{a c}=\alpha_{a} f^{m} I_{0} e^{-2 \alpha_{0} f^{m} z}
$$

where $\alpha_{a}$ is the frequency independent absorption coefficient which accounts for the energy that is absorbed and turned into heat. Absorption coefficient normally differs from attenuation coefficient because attenuation includes scattering as well. The difference is a proportionality constant $\left(\alpha_{a} / \alpha_{0}=a<=1\right.$, meaning absorption is always lower than attenuation). Because this proportionality constant does not change the optimum frequency calculation, we will use $a=1$.

The optimum frequency at which the power deposited at depth $z$ is maximum can be found by differentiating Eqn. 2 with respect to $f$ and equating it to zero. When we do that we obtain:

$$
f_{\text {opt }}=\left(\frac{1}{2 \alpha_{0} z}\right)^{(1 / m)}
$$

In soft tissue $m$ depends on the tissue type but typically can be approximated by 1 , in which case we arrive at the expression that relates application depth and frequency to attenuation coefficient:

$$
f_{\text {opt }} \cdot z=\frac{1}{2 \alpha_{0}}
$$

For example, in muscle, the frequency independent attenuation coefficient is equal to $\alpha_{0}=1.38 \times 10^{-7} \mathrm{~Np} /(\mathrm{Hz} \cdot \mathrm{cm})$ (approximately equal to $1 \mathrm{~dB} / \mathrm{MHz} / \mathrm{cm}$ ). Then. we find that $f_{\text {opt }} \times z=3.6 \mathrm{MHz} \cdot \mathrm{cm}$, which means, for example at $4 \mathrm{~cm}$ depth the optimum frequency is $900 \mathrm{kHz}$, and at $1 \mathrm{~cm}$ depth the optimum frequency is $3.6 \mathrm{MHz}$.

Although this calculation is done for plane waves and for homogenous medium, the results can be extended to focused transducers and inhomogeneous media. The details of these calculations can be found in [3] where it is shown that the simple expression of Eqn. 4 not only holds for focused waves but also can be extended to inhomogeneous media by replacing $\alpha_{0}$ with an average frequency independent attenuation coefficient. A FEM simulation study was also done showing results along the same line as well [4]. 

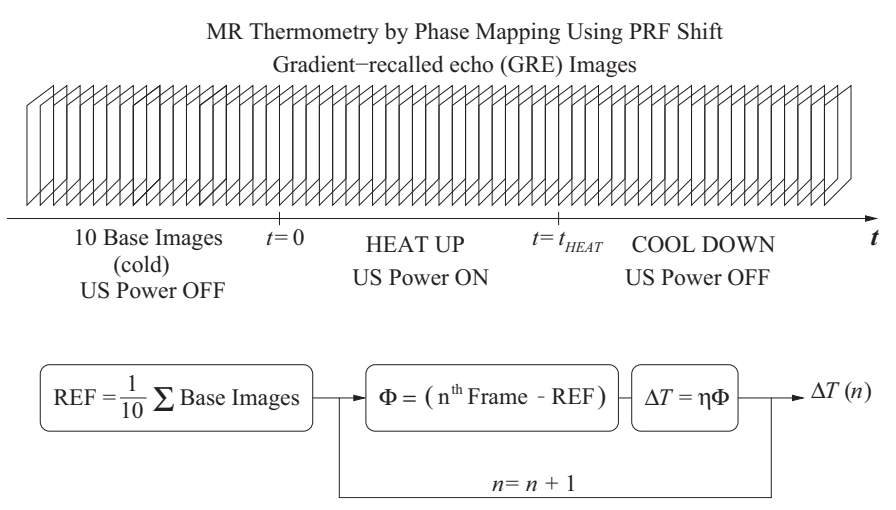

Fig. 1. Experimental Protocol

\section{EXPERIMENTAL METHODS}

In this study we used two MR compatible focused transducers that are designed and manufactured by Imasonic (Imasonic SAS, Voray sur l'Ognon, France): a $50 \mathrm{~mm}$ diameter $1 \mathrm{MHz}$ ( $40 \%$ fractional bandwidth) transducer with $50 \mathrm{~mm}$ fixed focus, and a $50 \mathrm{~mm}$ diameter $1.25 \mathrm{MHz}$ ( $40 \%$ fractional bandwidth) 16 element annular transducer array with $50 \mathrm{~mm}$ geometric focus. We calibrated these transducers using both a hydrophone (HNP-0400, ONDA Corp, Sunnyvale, CA, USA) and an ultrasound power meter (UPM-DT-10AV, Ohmic Instruments, St. Charles, MO, USA) with respect to frequency. We swept the frequency while keeping the acoustic power output of the transducers constant and measured the temperature increase in tissue using MR thermometry (Siemens Tim Trio 3T, Siemens AG, Erlangen, Germany).

We used the Phase Mapping by PRF Shift technique described in [5] for MR thermometry. In this method we continuously acquire Gradient Recalled Echo (GRE) images. After a number of base images (in this study we used 10 base images), the ultrasound (US) power is turned on $(t=0)$. After the heating duration $\left(t_{H E A T}\right.$ : varying from 30 seconds to 4 minutes), the US power is turned off and the sample is let cool down. GRE images are continuously acquired during this time (4 seconds/image for the sequence we used). The first 10 images are averaged and the result is used as a reference frame. The reference frame is then subtracted from the following frames to obtain the phase mapping of each frame (the images are complex valued images). Because the phase difference is proportional to the temperature differential, each phase difference image is converted to temperature map of the frame by multiplying by a constant. A schematic drawing of this protocol is shown in Fig. 1. This protocol is repeated for each US frequency by giving enough time (10 minutes) for the sample to cool down to room temperature.

We excited the fixed focus transducer with a signal generator (33521A Agilent, Santa Clara, CA, USA) followed by a broadband power amplifier (1040L E\&I, Rochester, NY, USA). We used a 16 channel pulse generator from IGT (IGT, France) to excite the annular array. As homogenous samples, we used lean ex-vivo cow muscle, cow liver and chicken muscle. We varied the sample thickness, acoustic power, and sonification duration as well as the frequency. We measured the temperature differential $(\Delta T)$, and temperature time gradient when the acoustic power is turned on $\left(\tau_{r}\right)$. Hence, we obtained

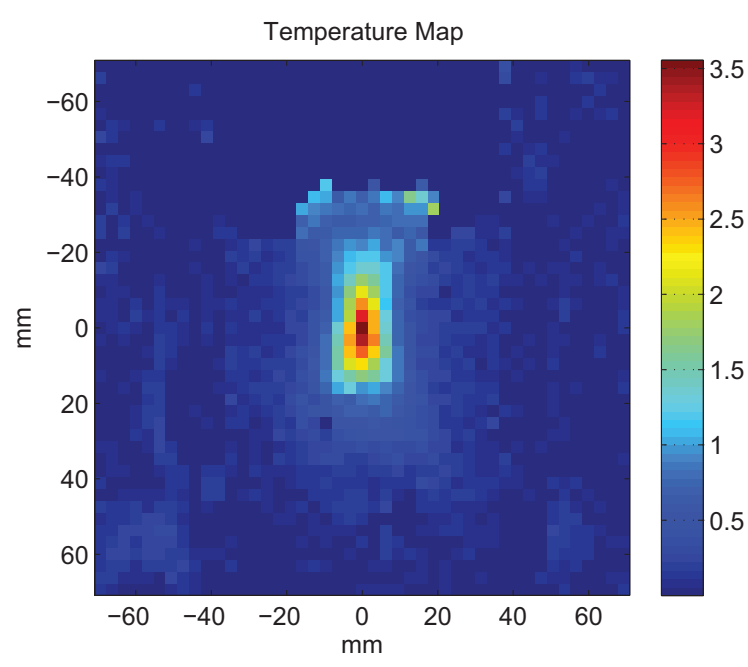

Fig. 2. Temperature map of the sample at $1400 \mathrm{kHz}$ at the end of the heat cycle.

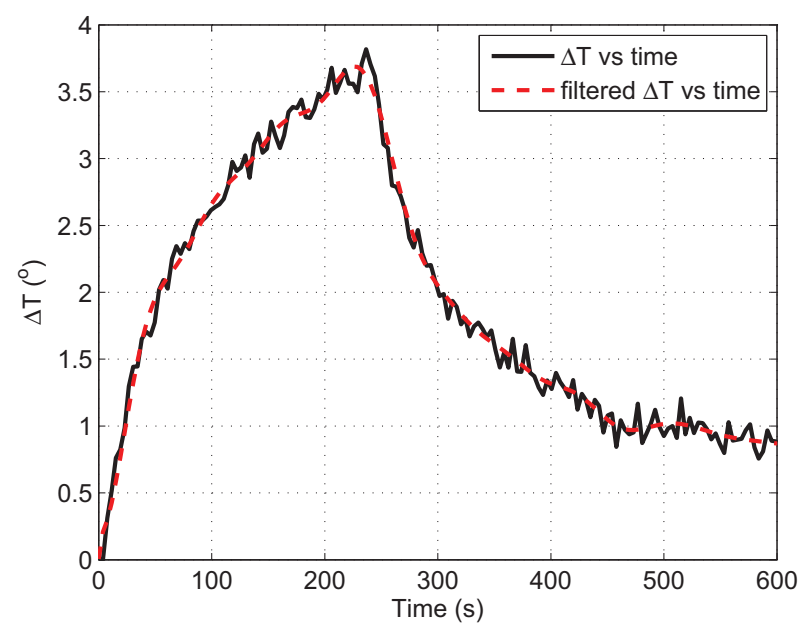

Fig. 3. Measured temperature differential at the focus of the transducer in chicken breast sample at $1400 \mathrm{kHz}$ : solid black line is measured data as is, and red dashed line is filtered measured data.

3 dimensional data sets $(x, y$, and $t)$ for each frequency and sample. As an example, Fig. 2 shows the temperature map of chicken breast sample at the end of the 4 min heat cycle for $1400 \mathrm{kHz}$ excitation. Each pixel on this map has a time axis as well. To reduce the noise in the temperature maps, we applied a simple FFT filtering in time domain to remove high frequency variations (we know that temperature variations cannot be of high frequency). Fig. 3 shows the actual measured data and its filtered version at the focus.

Another filtering method we used is to fit a linear/exponential rising function as shown in Eqn. 5 to the heat cycle of the data, where $c_{1}, c_{2}$ and $c_{3}$ are the fit parameters. This fit function is an expected consequence of the heat transfer problem and fits excellently to the data as shown in Fig. 4. We used the values at the end of the heat cycle from the linear/exponential fit to generate the plots in the next section.

$$
\Delta T(t)=\left(c_{1}+c_{2} \cdot t\right) \times\left(1-e^{-c_{3} \cdot t}\right)
$$




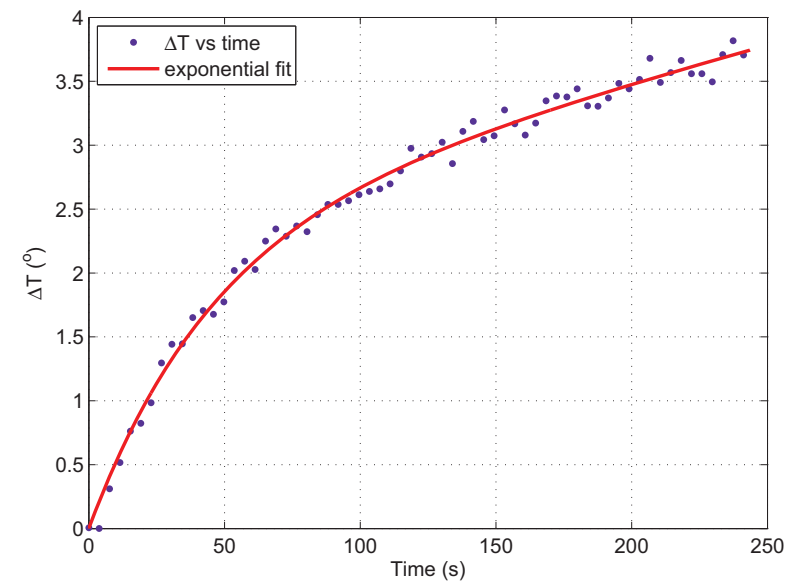

Fig. 4. Heat cycle of the temperature map at the focus of the transducer in chicken breast sample at $1400 \mathrm{kHz}$ : solid dots are the measured data as is, and red solid line is linear/exponential fit.

\section{EXPERIMENTAL Results}

1) Optimum frequency at different depths: For this experiment we used chicken breast samples, and the 16 channel MR compatible annular array together with the 16 channel beamformer/driver. We focused the transducer at $40 \mathrm{~mm}$ and swept the frequency, and repeated the experiment with the transducer focused at $70 \mathrm{~mm}$ using a fresh sample. We used 4 minute long continuous wave excitations with approximately $2 \mathrm{~W}$ of acoustic power, and measured the temperature change in the field of view. The peak temperature difference (temperature at the focus) at the end of the heat cycle is plotted in Fig. 5.

These two plots are quite informative. First of all, the 800 and $900 \mathrm{kHz}$ data points for the $40 \mathrm{~mm}$ and $70 \mathrm{~mm}$ data sets are identical. This is a nice observation of the rather obvious fact that the attenuation at these frequencies is so low that the acoustic intensity at different focal points is essentially the same resulting in the same temperature difference. Recall from Eqn. 2 that absorbed acoustic power is proportional to the acoustic intensity. Starting with $1 \mathrm{MHz}$ sample, the two data sets starts to deviate meaning that while the absorbed power at $40 \mathrm{~mm}$ depth continue to increase in a linear fashion with respect to frequency the attenuation starts to play its role at $70 \mathrm{~mm}$ depth.

Secondly, the $40 \mathrm{~mm}$ and $70 \mathrm{~mm}$ data sets were acquired on different days and with different samples. Obtaining the same temperature difference for the 800 and $900 \mathrm{kHz}$ data points on these two different data sets is an indication of the repeatability of the experiments.

There is little data on the acoustic attenuation coefficient of chicken breast in the literature. According to this limited data [6] the frequency independent attenuation coefficient $\alpha_{0}$ of chicken breast is approximately $5 \times 10^{-8} \mathrm{~Np} /(\mathrm{Hz} \cdot \mathrm{cm})$ which makes $f_{\text {opt }} \cdot z=10 \mathrm{Mhz} \cdot \mathrm{cm}$ according to Eqn. 4 . Hence, for $40 \mathrm{~mm}$ depth the expected optimum frequency is $2.5 \mathrm{Mhz}$, whereas for $70 \mathrm{~mm}$ depth the expected optimum frequency is $1.4 \mathrm{MHz}$. Before discussing how the experiments compare

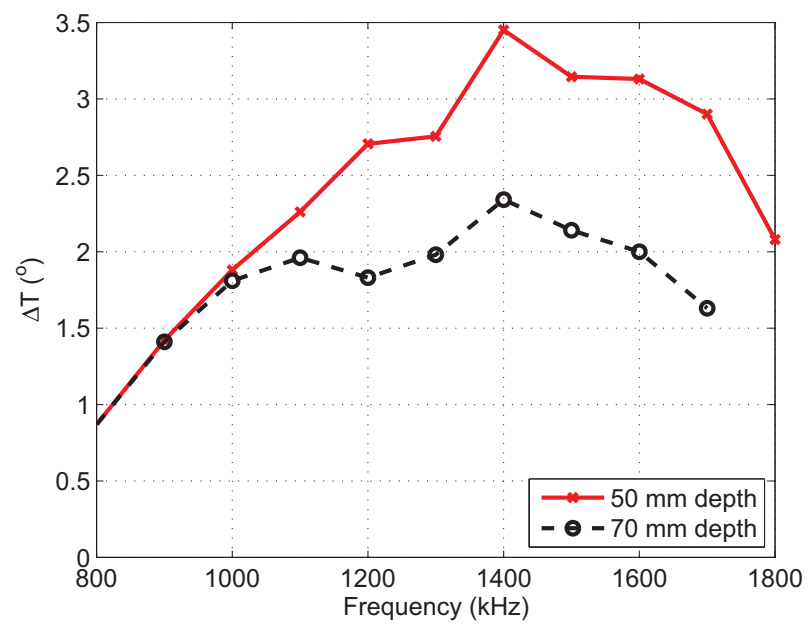

Fig. 5. Measured temperature differential at the focus of the transducer in chicken breast sample at $50 \mathrm{~mm}$ depth (dashed black line), and $66 \mathrm{~mm}$ depth (solid red line).

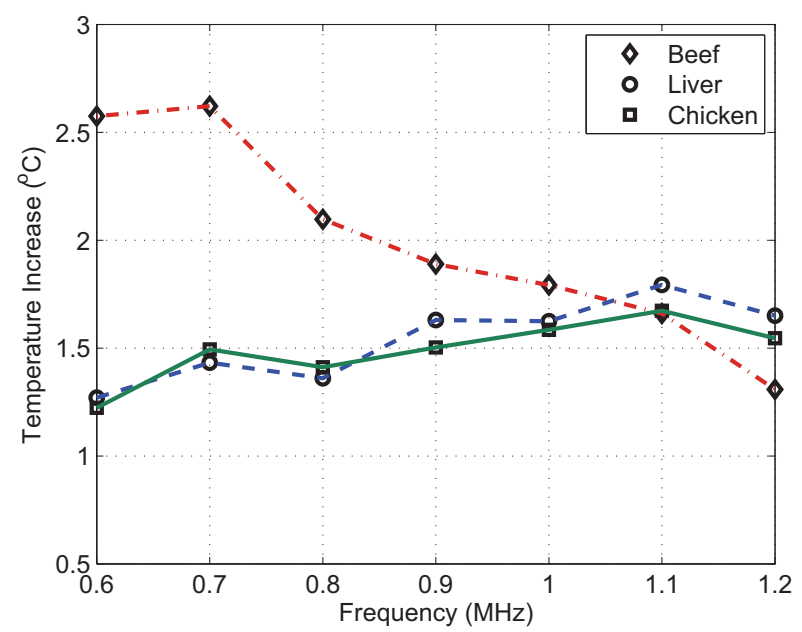

Fig. 6. Measured temperature differential at the focus of the transducer in muscle, liver and chicken breast samples.

to these expectations it should be noted that the somewhat irregular variations in the temperature vs. frequency graphs above $1.2 \mathrm{MHz}$ indicates that there is an inaccuracy in the acoustic output calibration of the annular transducer array at those frequencies. Because of this inaccuracy, fitting a smoothing curve over the data will likely give a better estimate of the optimum frequency: $1.3 \mathrm{MHz}$ and $1.6 \mathrm{MHz}$ for $70 \mathrm{~mm}$ and $40 \mathrm{~mm}$ depths, respectively.

2) Optimum frequency for different samples: For this experiment we used lean beef, liver and chicken breast samples, and the fixed focus $(50 \mathrm{~mm})$ MR compatible single element transducer together with a signal generater and power amplifier. We used 4 minute long continuous wave excitations with approximately $2 \mathrm{~W}$ of acoustic power, and measured the temperature change in the field of view. The peak temperature difference (temperature at the focus) at the end of the heat cycle for the three samples are plotted in Fig. 6. 
The frequency independent acoustic attenuation coefficient of lean beef, liver and chicken breast are $1.035 \times 10^{-7}$, $1.38 \times 10^{-7}$ and $5 \times 10^{-8}$, respectively. Consequently, the expected optimum frequencies for these three tissue types for a depth of $50 \mathrm{~mm}$ are $720 \mathrm{kHz}, 1.0 \mathrm{MHz}$, and $2.0 \mathrm{MHz}$, respectively. Although the irregular variations in the frequency response indicates to calibration errors, the curves indicate that for muscle and liver tissue the experimental results are close to expectations whereas for chicken breast sample the experimental optimum frequency is lower than expected.

\section{Discussion}

It is fair to say that the analytical optimum frequency expression of Eqn. 3 holds as a rule of thumb to determine the operating frequency for a particular HIFU application. MR thermometry results also revealed some nice observations predicted by analytical expressions. One of these observations is that the absorbed power (hence the temperature increase) is a slow varying function of frequency around the optimum. That means, the choice of operating frequency is not very critical as long as it is around the optimum. However, this is not always the case. The examples considered in this study only involve tissues with low attenuation coefficient. In case of very high attenuation coefficient samples such as intracranial applications, absorbed power (Eqn. 2) can be a rapidly varying function of frequency around the optimum because of the high attenuation in bone.

It should also be noted that the experimental results revealed some acoustic output power calibration inaccuracies at higher frequencies which turned out to be one of the most challenging tasks. With the calibrated hydrophone we measured the pressure at the focus of the transducer, backpropagated to the surface and converted the surface pressure to acoustic power. Locating the focus and back-propagating the pressure to the surface have been the source of inaccuracies in this method. With the ultrasound power meter that used radiation force balance method the measurements turned out to be more reliable than the hydrophone calibration. However, alignment between the ultrasound beam and the target turned out to be the source of inaccuracy in this case.

\section{CONCLUSION}

For HIFU applications (and acoustic radiation force application as well) there is a relationship between the optimum frequency and target depth determined by the average attenuation coefficient of the medium. The higher the attenuation coefficient the lower the optimum frequency gets. In this work, we conducted experiments in which we swept the HIFU frequency while keeping the acoustic power constant, and measured the temperature increase at the focus as a function of frequency. We repeated the experiments for varying focal depths and tissue types. The experimental results showed that the frequency at which the temperature increase is maximum is quite consistent with the expected optimum frequency for the focal depths and tissue samples evaluated in this study.

\section{ACKNOWLEDGMENT}

The authors would like to thank The Scientific and Technological Research Council of Turkey (TUBITAK) for the funding of this work (110E270 and 112E048), and National Magnetic Resonance Research Center (UMRAM) for their support.

\section{REFERENCES}

[1] D.N.Stephens, D.E. Kruse, A.S. Ergun, S. Barnes, X.M. Lu, K.W. Ferrara, Efficient Array Design for Sonotherapy, Phys in Med Biol, Vol. 53, pp. 3943-3969, 2008.

[2] C.R. Hill, Optimum Acoustic Frequency for Focused Ultrasound Surgery, Ultras in Med Biol, Vol. 20, No. 3, pp. 271-277, 1994.

[3] A.S. Ergun, Analytical and Numerical Calculations of Optimum Design Frequency for Focused Ultrasound Therapy and Acoustic Radiation Force, Ultrasonics, Vol. 51, pp. 786-794, 2011.

[4] A. Bozkurt, R.B. Roy, A.S. Ergun, Optimization of Operating Frequency of Acoustic Transducers for Obtaining Maximum Temperature in HIFU Based Therapeutic Ablation, IEEE Int Ultrasonics Symp., Oct 2012.

[5] V. Rieke, K.B. Pauly, MR Thermometry, J of Magn Res Im, Vol. 27, pp. 376-390, 2008

[6] P.D. Tyreus, C. Diederich, Two-dimensional acoustic attenuation mapping of high-temperature interstitial ultrasound lesions, Phys in Med Biol, Vol. 49, pp. 533-546, 2004.

[7] M.A. O'Reilly, A. Muller, and K. Hynynen Ultrasound insertion loss of rat parietal bone appears to be proportional to animal mass at submegahertz frequencies, Ultras in Med Biol Vol. 37 No. 11, pp. 19301937, 2011. 